\title{
FIBRATIONS OF LOCALLY SYMMETRIC SPACES AND THE FAILURE OF THE JORDAN-HÖLDER PROPERTY
}

\author{
F. E. A. JOHNSON
}

\begin{abstract}
We construct examples of locally symmetric spaces which fibre, with both base space and fibre also locally symmetric, in two distinct ways so that neither fibre nor base of one is homeomorphic to either fibre or base of the other. The simplest example is a complex surface which fibres over a complex curve in two essentially distinct ways.

In consequence, the Jordan-Hölder property fails for large classes of irreducible discrete subgroups of Lie groups.
\end{abstract}

0. Introduction. Let $\mathscr{C}$ be a class of groups. Recall that by a poly- $\mathscr{C}$ filtration on a group $\Gamma$ we mean a sequence $\left(\Gamma_{r}\right)_{0 \leqslant r \leqslant n}$ of subgroups of $\Gamma$ with $\{1\}=\Gamma_{0} \subset \Gamma_{1} \subset$ $\cdots \subset \Gamma_{n-1} \subset \Gamma_{n}=\Gamma, \Gamma_{r} \triangleleft \Gamma_{r+1}$, and $\Gamma_{r+1} / \Gamma_{r} \in \mathscr{C}$ for each $r$. A class $\mathscr{C}$ is said to possess the Jordan-Hölder property when any two poly- $\mathscr{C}$ filtrations $\left(\Gamma_{r}\right)_{0 \leqslant r \leqslant n}$, $\left(\Gamma_{s}^{\prime}\right)_{0 \leqslant s \leqslant m}$ on the same group $\Gamma$ are equivalent in the sense that

(i) $n=m$, and

(ii) $\Gamma_{\sigma(r)+1} / \Gamma_{\sigma(r)} \cong \Gamma_{r+1}^{\prime} / \Gamma_{r}^{\prime}$ for some bijection $\sigma:\{0, \ldots, n-1\} \rightarrow\{0, \ldots$, $n-1\}$.

In this paper, we show that certain geometrically important classes of groups fail to possess the Jordan-Hölder property. The classes in question are the following:

(i) FREE. The class of free groups of finite rank $\geqslant 2$.

(ii) SURFACE. The class of fundamental groups of closed surfaces of genus $\geqslant 2$.

(iii) $\mathscr{L}_{G}$. The class of torsion free irreducible cocompact lattices in a noncompact linear semisimple Lie group $G$ with finitely many connected components.

(iv) $\mathscr{A}_{\mathbf{G}}$. The class of torsion free arithmetic subgroups in a linear algebraic group $\mathbf{G}$, defined and almost simple over $\mathbf{Q}$, and such that $\left(\mathbf{G}_{\mathbf{R}}\right)_{0}$ has no compact simple factors.

Observe that SURFACE is just the class $\mathscr{L}_{\mathrm{PGL}_{2}(\mathbf{R})}$ and that FREE is just the class $\mathscr{A}_{\mathbf{P S L}_{2}(\mathbf{Q})}$.

Given a poly- $\mathscr{C}$ filtration $\left(\Gamma_{r}\right)_{0 \leqslant r \leqslant n}$, we call $n$ the height of the filtration. In fact, in each case, we show that the Jordan-Hölder property fails for groups of height 2. The results can be viewed geometrically, as providing examples of locally symmetric spaces which decompose as fibre bundles in which both base and fibre are locally

Received by the editors May 17, 1985.

1980 Mathematics Subject Classification: Primary 53C35, 20E15, 22E40; Secondary 55R10.

Key words and phrases. Locally symmetric space, irreducible discrete subgroup, Jordan-Hölder property. 
symmetric spaces in two essentially different ways, in the sense that neither base nor fibre of one fibration is either base or fibre of the other fibration. In the case of SURFACE, we construct a compact complex surface which fibres holomorphically over a complex curve in two essentially different ways.

1. A complex surface which fibres over a curve in two essentially distinct ways. Let $\Sigma^{+}(n)$ denote the closed orientable surface of genus $n$, and let $G(n)^{+}=\pi_{1}\left(\Sigma^{+}(n)\right)$. In all that follows, we shall take $n \geqslant 2$. $\Sigma^{+}(2 n-1)$ admits a fixed point free involution $T_{n}$ described as follows: Let $R(n-1)$ be the closed subset of $\mathbf{C}$ defined by

$$
\begin{aligned}
& R(n-1)=\left\{z \in \mathbf{C}:|z| \leqslant n-\frac{1}{2} \text { and } \frac{1}{3} \leqslant|z-r|,\right. \\
& \text { for all integers } r \text { with }|r| \leqslant n-1\} .
\end{aligned}
$$

$\Sigma^{+}(2 n-1)$ may be constructed as $R(n-1) \times\{0,1\} / \sim$ where $(w, 0) \sim(w, 1)$ for $w \in \partial R(n-1)$. The involution $\mathbf{C} \rightarrow \mathbf{C}, z \rightarrow-z$, maps $R(n-1)$ to itself without fixed points. Let $T_{n}: \Sigma^{+}(2 n-1) \rightarrow \Sigma^{+}(2 n-1)$ be the involution given by $T_{n}([z, i])$ $=[-z, i]$. Let $C_{2}$ be the cyclic group of order 2 , represented as $\left\{\mathrm{Id}, T_{n}\right\}$. Then it is straightforward to see that $C_{2} \backslash \Sigma^{+}(2 n-1)=\Sigma^{+}(n)$. Moreover, the covering map $\Sigma^{+}(2 n-1) \rightarrow \Sigma^{+}(n)$ imbeds $G(2 n-1)^{+}$in $G(n)^{+}$as a subgroup of index 2 .

Let $\Delta$ be the diagonal subgroup of $C_{2} \times C_{2}$;

$$
\Delta=\left\{(g, g): g \in C_{2}\right\} .
$$

Since $C_{2} \times C_{2}$, and hence $\Delta$, acts smoothly and freely on the smooth closed 4-manifold $\Sigma^{+}(2 n-1) \times \Sigma^{+}(2 m-1)$, then putting

$$
C(n, m)=\Delta \backslash\left(\Sigma^{+}(2 n-1) \times \Sigma^{+}(2 m-1)\right),
$$

we see that $C(n, m)$ is a smooth closed 4-manifold, which is doubly covered by $\Sigma^{+}(2 n-1) \times \Sigma^{+}(2 m-1)$, and which is itself a double cover of

$$
\Sigma^{+}(n) \times \Sigma^{+}(m)=Q \backslash C(n, m)
$$

where $Q=\left(C_{2} \times C_{2}\right) / \Delta$.

Let $c_{n}: \Sigma^{+}(2 n-1) \rightarrow \Sigma^{+}(n)$ be the covering map obtained by factoring out the $C_{2}$ action, and let $p_{1}: C(n, m) \rightarrow \Sigma^{+}(n)$ (resp. $p_{2}: C(n, m) \rightarrow \Sigma^{+}(m)$ ) be the map $p_{1}([w, z])=c_{n}(w)\left(\right.$ resp. $\left.p_{2}([w, z])=c_{m}(z)\right)$. Then it is easy to see that $p_{1}$ (resp. $\left.p_{2}\right)$ is a smooth fibre bundle with fibre $\Sigma^{+}(2 m-1)$ (resp. with fibre $\left.\Sigma^{+}(2 n-1)\right)$.

$$
\mathscr{F}_{1}=\left\{\begin{array}{ccc}
\Sigma^{+}(2 m-1) & \rightarrow & C(n, m) \\
& \downarrow \\
& \Sigma^{+}(n)
\end{array}, \quad \mathscr{F}_{2}=\left\{\begin{array}{ccc}
\Sigma^{+}(2 n-1) & C(n, m) \\
& \downarrow \\
& & \Sigma^{+}(m)
\end{array}\right.\right.
$$

Since all the spaces in question are aspherical, the long homotopy exact sequence of the fibration $\mathscr{F}_{i}$ reduces to a short exact sequence of groups $\mathscr{E}_{i}$, thus,

$$
\begin{aligned}
& \mathscr{E}_{1}=\left(1 \rightarrow G^{+}(2 m-1) \rightarrow \pi_{1}(C(n, m)) \rightarrow G^{+}(n) \rightarrow 1\right), \\
& \mathscr{E}_{2}=\left(1 \rightarrow G^{+}(2 n-1) \rightarrow \pi_{1}(C(n, m)) \rightarrow G^{+}(m) \rightarrow 1\right) .
\end{aligned}
$$

Hence, if $n \neq m$, then the poly-SURFACE filtrations on $\pi_{1}(C(n, m))$ are not Jordan-Hölder equivalent, and in addition we see that $C(n, m)$ fibres over a closed 2-manifold in two essentially different ways. Now $\Sigma^{+}(n)$ and $\Sigma^{+}(m)$ both admit 
(many) complex structures. Lifting these to $\Sigma^{+}(2 n-1)$ and $\Sigma^{+}(2 m-1)$, we see that $\Delta$ acts holomorphically on $\Sigma^{+}(2 n-1) \times \Sigma^{+}(2 m-1)$, inducing a complex structure on $C(n, m)$ in such a way that the projections onto $\Sigma^{+}(n)$ and $\Sigma^{+}(m)$ are holomorphic. Summarizing, we get

Proposition 1. If $n>m \geqslant 2$, then the complex surface $C(n, m)$ fibres holomorphically over a complex curve in two essentially different ways in the sense that neither base nor fibre of one fibration is either base or fibre of the other fibration.

Observing that SURFACE is the class of fundamental groups of real rather than complex surfaces, we get as a corollary the following

COROLlARY 2. SURFACE fails to have the Jordan-Hölder property.

2. Sufficient algebraic conditions for the failure of the Jordan-Hölder property. Clearly, a necessary condition for a class $\mathscr{C}$ of groups to have the Jordan-Hölder property is that if $\left(\Gamma_{r}\right)_{0 \leqslant r \leqslant n}$ and $\left(\Gamma_{s}^{\prime}\right)_{0 \leqslant s \leqslant m}$ are poly- $\mathscr{C}$ filtrations on the same group then $n=m$. We call this the weak Jordan-Hölder property.

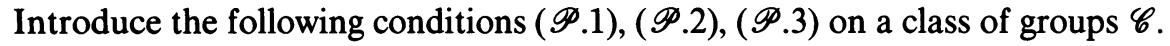

( $\mathscr{P} .1)$ For some fixed $n \geqslant 1$, each $\Gamma \in \mathscr{C}$ is a duality group ([1]) of dimension $n$.

( $\mathscr{P} .2)$ For each $\Delta \in \mathscr{C}$, if $\Gamma \subset \Delta$ is a subgroup of finite index then $\Gamma \in \mathscr{C}$ and, moreover, $\Delta \cong \Gamma$ iff $\Delta=\Gamma$.

( $\mathscr{P} .3)$ For each $\Delta \in \mathscr{C}$ there is a prime $q$ and an epimorphism $\Gamma \rightarrow Q(q)$, where $Q(q)$ is a finite group of order $q^{n}$, for some $n \geqslant 2$, and $\Gamma$ is a subgroup of finite index in $\Delta$.

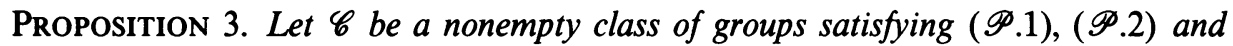
(PP.3). Then $\mathscr{C}$ has the weak Jordan-Hölder property, but fails to have the Jordan-Hölder property.

Proof. In fact, the weak Jordan-Hölder property is guaranteed by (P्P.1). For if $\left(\Gamma_{r}\right)_{0 \leqslant r \leqslant k}$ is a poly- $\mathscr{C}$ filtration on $\Gamma=\Gamma_{k}$, then since $\Gamma_{r+1} / \Gamma_{r}$ is a duality group of dimension $n, \Gamma$ is a duality group of dimension $k n$. Thus if $\left(\Gamma_{r}^{\prime}\right)_{0 \leqslant r \leqslant m}$ is another poly- $\mathscr{C}$ filtration on $\Gamma$, then $m n=k n$, so that $m=k$, as claimed.

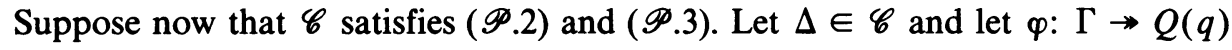
be an epimorphism onto a group $Q(q)$ with $q^{n}$ elements, $n \geqslant 2$, and where $\Gamma$ is a subgroup of finite index in $\Delta$. Let $\pi_{0}: Q(q) \rightarrow \mathrm{Z} / q \mathrm{Z}$ be an epimorphism, which exists since $Q(q)$ is nilpotent. Moreover, $\operatorname{Ker}\left(\pi_{0}\right)$ has order $q^{n-1}$, so we may choose an epimorphism $\pi_{1}: \operatorname{Ker}\left(\pi_{0}\right) \rightarrow \mathbf{Z} / q \mathbf{Z}$. Put $\Gamma_{0}=\Gamma, \Gamma_{1}=\varphi^{-1}\left(\operatorname{Ker}\left(\pi_{0}\right)\right)$, and form the fibre product

$$
\Psi=\Gamma_{0} \times \Gamma_{1}
$$

where $f=\pi_{0} \varphi$ and $g=\pi_{1} \varphi: \Gamma_{1} \rightarrow \mathbf{Z} / q \mathbf{Z}$. Let $\Gamma_{2}=\operatorname{Ker}(g) \subset \Gamma_{1}$. If $p_{r}: \Psi \rightarrow \Gamma_{r}$ is the projection map for $r=0,1$, and if $i_{0}, i_{1}$ are the injective maps $i_{0}: \Gamma_{2} \rightarrow \Psi$, $i_{0}(y)=(1, y)$ and $i_{1}: \Gamma_{1} \rightarrow \Psi, i_{1}(x)=(x, 1)$, then $\operatorname{Ker}\left(p_{r}\right)=\operatorname{Im}\left(i_{r}\right)$ for $r=0,1$, 
and we have a pair of exact sequences

$$
\begin{aligned}
& \mathscr{E}_{0}=\left(1 \rightarrow \Gamma_{2} \stackrel{i_{0}}{\rightarrow} \Psi \stackrel{p_{0}}{\rightarrow} \Gamma_{0} \rightarrow 1\right), \\
& \mathscr{E}_{1}=\left(1 \rightarrow \Gamma_{1} \stackrel{i_{1}}{\rightarrow} \Psi \stackrel{p_{1}}{\rightarrow} \Gamma_{1} \rightarrow 1\right) .
\end{aligned}
$$

Since $\Gamma_{i+1}$ is a proper subgroup of finite index in $\Gamma_{i}$, then $\Gamma_{i+1} \not \Gamma_{i}$ for $i=0,1$, so that $\mathscr{E}_{0}, \mathscr{E}_{1}$ define a pair of poly- $\mathscr{C}$ filtrations on $\Psi$ which are not Jordan-Hölder equivalent. Q.E.D.

3. Lattice groups and locally symmetric spaces. In this section we observe that if $G$ is a noncompact linear semisimple Lie group with finitely many components, then $\mathscr{L}_{G}$, the class of torsion free irreducible cocompact lattices in $G$, satisfies ( $\left.\mathscr{P} .1\right)$, $(\mathscr{P} .2)$ and (PP.3), and thus fails to have the Jordan-Hölder property. We also construct examples of locally symmetric spaces which fibre over locally symmetric spaces in more than one way. Since SURFACE $=\mathscr{L}_{\mathrm{PGL}_{2}(\mathbf{R})}$, this generalises the construction of $\S 1$.

Similarly, if $\mathbf{G}$ is a linear algebraic group defined and almost simple over $\mathbf{Q}$ such that $\mathbf{G}_{\mathbf{R}}$ is noncompact, then $\mathscr{A}_{\mathbf{G}}$, the class of torsion free arithmetic subgroups in G, satisfies $(\mathscr{P} .1),(\mathscr{P} .2)$ and $(\mathscr{P} .3)$.

Proposition 4. Let $G$ be a noncompact linear semisimple Lie group having only

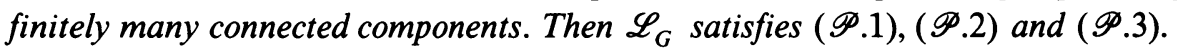

Proof. In fact, if $K$ is a maximal compact subgroup of $G$, then $G / K$ is diffeomorphic to some $\mathbf{R}^{n}$ [7], and if $\Gamma \in \mathscr{L}_{G}$, then since $\Gamma$ is torsion free, $\Gamma \backslash G / K$ is a smooth closed manifold of type $K(\Gamma, 1)$. Hence $\Gamma$ is actually a Poincaré Duality group [6] of dimension $n$, thus verifying (PP.1).

Now if $\Delta \in \mathscr{C}$ then $\Delta$ is a finitely generated linear group and hence there are infinitely many primes $q$ such that $\Delta$ is virtually a residually-\{finite $q$-group\}. See, for example, [8, p. 55, Theorem 4.7]. Choosing one such prime $q$, there is a subgroup $\Gamma$ of finite index in $\Delta$ and an epimorphism $\Gamma \rightarrow Q(q)$, where $Q(q)$ has order $q^{n}$ for

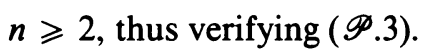

Let $\Delta \in \mathscr{L}_{G}$ and $\Gamma \subset \Delta$ be a subgroup of finite index in $\Delta$. Then it is clear that $\Gamma$ is still an irreducible torsion free cocompact discrete subgroup of $G$, hence $\Gamma \in \mathscr{L}_{G}$. Now suppose $\Gamma \cong \Delta$. We must show that $\Gamma=\Delta$.

Let $G_{0}$ be the identity component of $G$, and let $C$ be a maximal connected compact normal subgroup of $G_{0}$. Observe that $C$ is characteristic in $G_{0}$, hence normal in $G$, and that, since $\Delta$ is torsion free, $\Delta$ imbeds as an irreducible cocompact lattice in $G / C$. We distinguish two cases, according to whether $G_{0} / C$ is locally isomorphic to $\mathrm{PSL}_{2}(\mathbf{R})$ or not. If $G_{0} / C$ is not locally isomorphic to $\mathrm{PSL}_{2}(\mathbf{R})$, then since $\Delta$ is irreducible in $G_{0} / C$, the equality $\Gamma=\Delta$ follows from a theorem of Borel [2, Theorem 1.7]. If $G_{0} / C$ is locally isomorphic to $\operatorname{PSL}_{2}(\mathbf{R})$, then $\Delta$ is the fundamental group of the closed surface $\Delta \backslash G / K$ where $K$ is a maximal compact subgroup of $G$. Moreover, $\Delta \backslash G / K$ is not homeomorphic to either $S^{2}, \mathbf{R P}^{2}, S^{1} \times S^{1}$, or the Klein bottle $\left(S^{1} \times S^{1}\right) \# \mathbf{R} \mathbf{P}^{2}$. In particular, the Euler characteristic $\chi(\Delta \backslash G / K)$ is 
nonzero, and depends only upon the isomorphism type of $\Delta$, since $\Delta \backslash G / K$ is a complex of type $K(\Delta, 1)$, and we may write $\chi(\Delta)=\chi(\Delta \backslash G / K)$. Now $\Gamma \backslash G / K$ is an $n$-fold covering of $\Delta \backslash G / K$ where $n$ is the index of $\Gamma$ in $\Delta$. Hence $\chi(\Gamma)=n \chi(\Delta)$. However, since $\Gamma \cong \Delta$, then $\chi(\Gamma)=\chi(\Delta)$, so that $n=1$, and $\Gamma=\Delta$. Q.E.D.

We should observe that the hypothesis of irreducibility of $\Gamma$ in $G$ forces the class $\mathscr{L}_{G}$ to be empty in many cases. In fact, using the notation of the previous proof, if $H=G / C$, then projection of $G$ onto $H$ induces an inclusion $\mathscr{L}_{G} \subset \mathscr{L}_{H}$. Of course, if $H_{0}$ is almost simple, then $\mathscr{L}_{H} \neq \varnothing$, since the hypothesis of irreducibility is trivially satisfied. Let $\mathfrak{h}$ be the real Lie algebra of $H_{0}$, and let

$$
\mathfrak{h} \otimes \mathbf{C} \cong a_{1} \oplus \cdots \oplus a_{m}
$$

be the decomposition of $\mathfrak{h} \oplus \mathbf{C}$ into simple ideals $\mathfrak{a}_{i}$. A necessary and sufficient condition for $\mathscr{L}_{H} \neq \varnothing$ (and thus for $\mathscr{L}_{G} \neq \varnothing$ also) is that $\mathfrak{h} \otimes \mathbf{C}$ be isotypic, i.e. that $\mathfrak{a}_{1} \cong \mathfrak{a}_{2} \cong \cdots \cong \mathfrak{a}_{m}$. See, for example, [5]. In particular, $\mathscr{L}_{\mathrm{SL}_{2}(\mathbf{R}) \times \mathrm{SL}_{3}(\mathbf{R})}=\varnothing$, although $\mathscr{L}_{\mathrm{SL}_{2}(\mathbf{R}) \times \mathrm{SL}_{2}(\mathbf{R})} \neq \varnothing$.

Proposition 5. Let $\mathbf{G}$ be a linear algebraic group defined and almost simple over $\mathbf{Q}$, and such that $\left(\mathbf{G}_{\mathbf{R}}\right)_{0}$ has no compact simple factor. Then $\mathscr{A}_{\mathbf{G}}$ satisfies $(\mathscr{P} .1),(\mathscr{P} .2)$ and $(\mathscr{P} .3)$.

Proof. If $\Gamma \in \mathscr{A}_{\mathbf{G}}$ then by a theorem of Borel and Serre [3, 11.4.2], $\Gamma$ is a duality group of dimension $d-r$, where $d=\operatorname{dim}\left(\mathbf{G}_{\mathrm{R}} / K\right), K$ a maximal compact subgroup of $\mathbf{G}_{\mathbf{R}}$, and $r=\operatorname{rank}_{\mathbf{Q}}(\mathbf{G})$. Thus $\mathscr{A}_{\mathbf{G}}$ satisfies $(\mathscr{P} .1)$.

In particular, each $\Gamma \in \mathscr{A}_{\mathbf{G}}$ is a finitely generated linear group, so the proof of

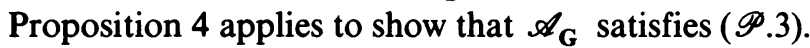

If $\Delta \in \mathscr{A}_{\mathbf{G}}$ and $\Gamma \subset \Delta$ is a subgroup of finite index, then $\Gamma \in \mathscr{A}_{\mathbf{G}}$. If, in addition, $\Gamma \cong \Delta$ we must show that $\Gamma=\Delta$. Since $\mathbf{G}$ is almost $\mathbf{Q}$-simple and $\left(\mathbf{G}_{\mathbf{R}}\right)_{0}$ has no compact factors, then Theorem (2.2) of [5] applies to show that $\Delta$ is irreducible in $\mathbf{G}_{\mathbf{R}}$. If $\Delta$ is cocompact in $\mathbf{G}_{\mathbf{R}}$ then we may apply Proposition 4 to conclude that $\Gamma=\Delta$.

If $\Delta \backslash \mathbf{G}_{\mathbf{R}}$ is noncompact, we distinguish two cases:

(i) $\left(G_{R_{R}}\right)_{0}$ is not locally isomorphic to $\operatorname{PSL}_{2}(R)$,

(ii) $\left(\mathbf{G}_{\mathbf{R}}\right)_{0}$ is locally isomorphic to $\mathrm{PSL}_{2}(\mathbf{R})$.

In case (i), $\Gamma=\Delta$ by a theorem of Borel [2, (1.7)].

In case (ii), $\Delta$ is a free group $F_{n}$ of rank $n \geqslant 2$, and $\Gamma$, being a subgroup of finite index $d$ in $\Delta$, is a free group $F_{m}$ of rank $m$. By the Riemann-Hurwitz formula or, equivalently, using the Euler characteristic argument of Proposition 4, we get

$$
(m-1)=d(n-1) \text {. }
$$

Hence $m=n+(d-1)(n-1)$. Since $\Gamma \cong \Delta$, then $m=n$, so that, since $n \neq 1$, $d=1$ and $\Gamma=\Delta$. Q.E.D.

The geometric construction of $\S 1$ may be generalised as follows: Let $G$ be some suitable noncompact linear semisimple Lie group admitting an irreducible cocompact lattice, and let $X=G / K$ be the associated symmetric space. Choose $\Gamma_{0} \in \mathscr{L}_{G}$ such that $\Gamma_{0}$ maps onto some $Q(q)$ of order $q^{n}$ for some prime $q$, and some $n \geqslant 2$. Choose epimorphisms $\pi_{0}: \Gamma_{0} \rightarrow \mathbf{Z} / q \mathbf{Z}$ and $\pi_{1}: \Gamma_{1} \rightarrow \mathbf{Z} / q \mathbf{Z}$ where $\pi_{1}=\operatorname{Ker}\left(\pi_{0}\right)$. 
Put $\Gamma_{2}=\operatorname{Ker}\left(\pi_{1}\right)$, and let $X_{i}=\Gamma_{i} \backslash X$. If $\varphi$ is an epimorphism $\varphi: \Gamma_{0} \rightarrow Q(q)$ put $f=\pi_{0} \varphi, g=\pi_{1} \varphi$, and form the fibre product

$$
\Psi=\Gamma_{0} \times \Gamma_{f, g} .
$$

$\Psi$ acts freely on $X \times X$ by means of $\left(\gamma_{0}, \gamma_{1}\right) \cdot(x, y)=\left(\gamma_{0} \cdot x, \gamma_{1} \cdot y\right)$. Putting $Y=\Psi \backslash(X \times X)$, we have a pair of inequivalent fibre bundles, where $p_{i}$ is projection onto $X_{i}$.

$$
\mathscr{F}_{1}=\left\{\begin{array}{cccc}
X_{2} & \hookrightarrow & Y \\
& & \downarrow p_{0}, \\
& X_{0}
\end{array} \quad \mathscr{F}_{2}=\left\{\begin{array}{ccc}
X_{1} & \hookrightarrow & Y \\
& & \downarrow p_{1} \\
& & X_{1}
\end{array}\right.\right.
$$

There is an analogous construction using $\Gamma_{0} \in \mathscr{A}_{\mathbf{G}}$, although in this case the locally symmetric spaces are generally noncompact.

4. Some special cases of the Jordan-Hölder property. Let $\mathfrak{L}=\{H: H$ is a discrete cocompact subgroup in a noncompact, connected simple adjoint Lie group $L$ with $\left.\operatorname{rank}_{\mathbf{R}}(L) \geqslant 2\right\}$. In [4] we showed [4, (2.1) and (2.8)] that if $\Gamma, \Gamma^{\prime}$ are finite products

$$
\begin{aligned}
& \Gamma \cong C_{1}^{\left(e_{1}\right)} \times \cdots \times C_{m}^{\left(e_{m}\right)}, \\
& \Gamma^{\prime} \cong D_{1}^{\left(f_{1}\right)} \times \cdots \times D_{n}^{\left(f_{n}\right)}
\end{aligned}
$$

with $C_{i}, D_{j} \in \mathfrak{R} \cup$ FREE $\cup$ SURFACE, $e_{i}, f_{j}>0$, and $C_{i} \neq C_{j}$ if $i \neq j, D_{r} \neq D_{s}$ if $r \neq s$. Then $\Gamma \cong \Gamma^{\prime} \Rightarrow(m=n) \&\left(D_{\sigma(i)} \cong C_{i}\right.$, and $f_{\sigma(i)}=e_{i}$ for some bijection $\sigma$ : $\{1, \ldots, m\} \rightarrow\{1, \ldots, m\})$. Thus we have a Jordan-Hölder theorem for such products.

In fact, the proof of this result has a wider range of validity. For example, it is not difficult to show that a similar result holds if $\mathfrak{L} \cup$ FREE $\cup$ SURFACE is replaced by

(i) $\mathscr{L}_{G}$ for any linear semisimple Lie group with finitely many components,

(ii) $\bigcup_{G \in \mathscr{C}} \mathscr{L}_{G}$ where $\mathscr{C}$ is any class of groups $G$ as in (i), such that $\mathscr{C}$ contains at most one group $G$ with $\operatorname{rank}_{\mathbf{R}}\left(G_{0}\right)=1$,

(iii) $\cup_{\mathbf{G} \in \mathscr{D}} \mathscr{A}_{\mathbf{G}}$ where $\mathscr{D}$ is any class of linear algebraic groups defined and almost simple over $\mathbf{Q}$, containing at most one group $\mathbf{G}$ with $\operatorname{rank}_{\mathbf{R}}\left(\left(\mathbf{G}_{\mathbf{R}}\right)_{0}\right)=1$.

We began this paper with the intention of investigating whether the Jordan-Hölder property holds in a more general setting. Observe that in using lattices in Lie groups as "building blocks," one requires some form of irreducibility at the outset, even to obtain a Jordan-Hölder theorem for products. If nontrivial direct products are allowed as building blocks, the Jordan-Hölder property fails immediately. On the positive side one has, for example, the following

PROPOSITION 6. Let $\left(\Gamma_{r}\right)_{0 \leqslant r \leqslant m}$ be a poly-FREE filtration on $\Gamma=\Gamma_{m}$, and suppose that $p_{1}, \ldots, p_{m}$ are distinct primes such that $\Gamma_{r} / \Gamma_{r-1} \cong F_{p_{r}+1}$, the free group of rank $p_{r}+1$. Then any other poly-FREE filtration on $\Gamma$ is Jordan-Hölder equivalent to $\left(\Gamma_{r}\right)_{0 \leqslant r \leqslant m}$. 
ProOF. If ( $1 \rightarrow K \nrightarrow G \rightarrow Q \rightarrow 1$ ) is an extension of finitely presented groups of type FL, then the Euler characteristic behaves multiplicatively; thus,

$$
\chi(G)=\chi(K) \chi(Q)
$$

Thus we get

$$
\begin{aligned}
\chi(\Gamma) & =\chi\left(F_{p_{1}+1}\right) \cdots \chi\left(F_{p_{m}+1}\right) \\
& =(-1)^{m} p_{1} \cdots p_{m} .
\end{aligned}
$$

Hence the result follows from uniqueness of prime factorization. Q.E.D.

There is a similar result to the above using orientable SURFACE groups as composition factors, and genus instead of rank.

\section{REFERENCES}

1. R. Bieri and B. Eckmann, Groups with homological duality generalising Poincaré duality, Invent. Math. 20 (1973), 103-124.

2. A. Borel, On the automorphisms of certain subgroups of semisimple Lie groups, Algebraic Geometry, Bombay Colloquium, Oxford Univ. Press, London, 1969, pp. 43-73.

3. A. Borel and J. P. Serre, Corners and arithmetic groups, Comment. Math. Helv. 48 (1974), 244-297.

4. F. E. A. Johnson, Automorphisms of direct products of groups and their geometric realisations, Math. Ann. 263 (1983), 343-364.

5. __ On the existence of irreducible lattices, Arch. Math. 43 (1984), 391-396.

6. F. E. A. Johnson and C. T. C. Wall, On groups satisfying Poincaré duality, Ann. of Math. 96 (1972), 592-598.

7. G. D. Mostow, Selfadjoint groups, Ann. of Math. 62 (1955), 44-55.

8. B. A. F. Wehrfritz, Infinite linear groups, Ergeb. Math. Grenzgeb., vol. 76, Springer-Verlag, Berlin and New York, 1973.

Department of Mathematics, University College London, Gower Street, London WC1E 6BT, ENGLAND 